\title{
Prevalência de achados histopatológicos de mucosa esofágica e sua correlação com Helicobacter pylori
}

Prevalence of histopathological findings of esophageal mucosa and their correlation with Helicobacter pylori

Prevalencia de hallazgos histopatológicos de mucosa esofágica y su mantenimiento con Helicobacter pylori

Gabrielle Barbosa Vasconcelos de Souza ORCID: https://orcid.org/0000-0003-2465-356X Universidade Tiradentes, Brasil

E-mail: gabriellebrbsa@gmail.com

Elomar Rezende Moura

ORCID: https://orcid.org/0000-0002-1232-0146 Universidade Tiradentes, Brasil

E-mail: elomar.rezende@ souunit.com.br

Yasmin Tourinho Delmondes Trindade ORCID: https://orcid.org/0000-0003-3054-9187 Universidade Tiradentes, Brasil

E-mail: yasmindelmondes@gmail.com

Durval José de Santana Neto ORCID: https://orcid.org/0000-0002-5730-5118 Universidade Tiradentes, Brasil

E-mail: durvalneto777@gmail.com

Larissa Gonçalves Moreira ORCID: https://orcid.org/0000-0002-5968-8634 Universidade Tiradentes, Brasil

E-mail: larigoncalvesm@gmail.com

Gabriel Ponciano Santos de Carvalho ORCID: https://orcid.org/0000-0001-7476-7907 Universidade Tiradentes, Brasil E-mail: gabriel.ponciano19@gmail.com

Íkaro Daniel de Carvalho Barreto ORCID: https://orcid.org/0000-0001-7253-806X

Universidade Federal de Pernambuco, Brasil

E-mail: daniel.carvalho.ib@gmail.com Décio Fragata da Silva ORCID: https://orcid.org/0000-0003-1157-6198 Universidade Tiradentes, Brasil E-mail: dfragata@yahoo.com.br Luíse Meurer

ORCID: https://orcid.org/0000-0002-1315-4899 Laboratório de Patologia Monica Araújo, Brasil E-mail: luisemeurer@gmail.com

Leda Maria Delmondes Freitas Trindade ORCID: https://orcid.org/0000-0003-4300-4274 Universidade Tiradentes, Brasil E-mail: ledeltrin@gmail.com

\section{Resumo}

Introdução: patologias esofágicas com alterações histopatológicas podem provocar sintomas dispépticos, dor retroesternal e epigástrica. Objetivos: identificar a prevalência de achados histopatológicos de mucosa esofágica e correlacionar com a bactéria Helicobacter pylori (HP). Metodologia: estudo transversal, retrospectivo, com 1.953 laudos histopatológicos de mucosa esofágica. Dados analisados por frequência absoluta e relativa percentual, testes QuiQuadrado de Pearson, Mann-Whitney e Teste Qui-Quadrado com simulações de Monte-Carlo. Resultados: Analisaramse 1953 laudos de lesões esofágicas: sexo feminino $982(50,3 \%)$ e idade mediana 44. Identificou-se $151(7,7 \%)$ laudos positivos para HP e destes, 41 (2,3\%) tinham atrofia gástrica. A esofagite $1427(73,1 \%)$, a metaplasia $548(28,1 \%)$ e o esôfago de Barrett 133 (6,8\%) foram mais prevalentes. Malignidade, 5 (0,3\%) laudos. Dos portadores de HP: esofagite 
97 (64,2\%), esôfago de Barrett $17(11,3 \%)$ e metaplasia $45(29,8 \%)$ - metaplasia pancreática estatisticamente significativa $(\mathrm{p}<0,001)$. Dois $(1,3 \%)$ laudos apresentaram malignidade associados à HP. Correlacionando HP com lesões esofágicas, observou-se tendência a determinar maior risco da presença da bactéria: esôfago de Barret (RR: 1,68 (IC95\%: 1,05-2,69), hiperplasia epitelial (RR: 2,18 (IC95\%: 1,18-4,02), acantose glicogênica (RR: 3,69 (IC95\%: 2,086,54), pólipos (RR: 2,17 (IC95\%: 1,14-4,16), malignidade (RR: 4,91 (IC95\%: 1,66-14,52) e atrofia gástrica (RR: 3,44 (IC95\%: 2,02-5,84). Conclusão: Esôfago de Barrett, hiperplasia epitelial, acantose glicogênica, pólipos, malignidade e atrofia gástrica estão associados a um maior risco da presença da bactéria.

Palavras-chave: Mucosa esofágica; Histopatologia; Helicobacter pylori.

\begin{abstract}
Introduction: esophageal pathologies with histopathological changes can cause dyspeptic symptoms, retrosternal and epigastric pain. Objectives: to identify the prevalence of histopathological findings of the esophageal mucosa and to correlate with the bacterium Helicobacter pylori (HP). Methodology: cross-sectional, retrospective study, with 1,953 histopathological reports of the esophageal mucosa. Data analyzed by absolute and relative frequency, Pearson's ChiSquare, Mann-Whitney and Chi-Square Test with Monte-Carlo simulations. Results: 1953 reports of esophageal lesions were analyzed: female gender $982(50.3 \%)$ and median age 44. $151(7.7 \%)$ reports were positive for HP and of these, $41(2.3 \%)$ had gastric atrophy. Esophagitis 1427 (73.1\%), metaplasia 548 (28.1\%) and Barrett's esophagus $133(6.8 \%)$ were more prevalent. Malignancy, $5(0.3 \%)$ reports. Of the patients with PH: esophagitis $97(64.2 \%)$, Barrett's esophagus $17(11.3 \%)$ and metaplasia $45(29.8 \%)$ - statistically significant pancreatic metaplasia (p<0.001). Two (1.3\%) reports showed malignancy associated with PH. Correlating HP with esophageal lesions, there was a tendency to determine a higher risk of the presence of the bacteria: Barrett's esophagus (RR: 1.68 (95\%CI: 1.05-2.69), epithelial hyperplasia (RR: 2.18 (RR: 2.18) 95\%CI: 1.18-4.02), glycogenic acanthosis (RR: 3.69 (95\%CI: 2.08-6.54), polyps (RR: 2.17 (95\%CI: 1.14-4, 16), malignancy (RR: 4.91 (95\%CI: 1.66-14.52) and gastric atrophy (RR: 3.44 (95\%CI: $2.02-$ 5.84). Conclusion: Barrett's esophagus, Epithelial hyperplasia, glycogenic acanthosis, polyps, malignancy and gastric atrophy are associated with an increased risk of the presence of the bacteria.
\end{abstract}

Keywords: Esophageal mucosa; Histopathology; Helicobacter pylori.

\title{
Resumen
}

Introducción: las patologías esofágicas con cambios histopatológicos pueden causar síntomas dispépticos, dolor retroesternal y epigástrico. Objetivos: identificar la prevalencia de hallazgos histopatológicos de la mucosa esofágica y correlacionarlos con la bacteria Helicobacter pylori (HP). Metodología: estudio transversal, retrospectivo, con 1.953 informes histopatológicos de la mucosa esofágica. Datos analizados por frecuencia absoluta y relativa, Chi-Cuadrado de Pearson, Mann-Whitney y Chi-Cuadrado Test con simulaciones Monte-Carlo. Resultados: Se analizaron 1953 reportes de lesiones esofágicas: sexo femenino 982 (50,3\%) y mediana de edad $44.151(7,7 \%)$ reportes fueron positivos para HP y de estos, $41(2,3 \%)$ presentaron atrofia gástrica. La esofagitis $1427(73,1 \%)$, la metaplasia $548(28,1 \%)$ y el esófago de Barrett 133 (6,8\%) fueron los más prevalentes. Malignidad, 5 (0,3\%) informes. De los pacientes con HP: esofagitis 97 (64,2\%), esófago de Barrett 17 (11,3\%) y metaplasia 45 (29,8\%) - metaplasia pancreática estadísticamente significativa $(\mathrm{p}<0,001)$. Dos $(1,3 \%)$ informes mostraron malignidad asociada con HP. Al correlacionar HP con lesiones esofágicas, hubo una tendencia a determinar un mayor riesgo de presencia de las bacterias: esófago de Barrett (RR: 1,68 (IC 95\%: 1,05-2,69), hiperplasia epitelial (RR: 2,18 (RR: 2,18) $95 \%$ IC: 1,18-4,02), acantosis glucogénica (RR: 3,69 (95\%IC: 2,08-6,54), pólipos (RR: 2,17 (95\%IC: 1,14-4, 16), malignidad (RR: 4,91 (95\%IC : 1,66-14,52) y atrofia gástrica (RR: 3,44 (IC 95\%: 2,02-5,84). Conclusión: el esófago de Barrett, la hiperplasia epitelial, la acantosis glucogénica, los pólipos, la malignidad y la atrofia gástrica se asocian con un mayor riesgo de presencia de la bacteria. Palabras clave: Mucosa esofágica; Histopatología; Helicobacter pylori.

\section{Introdução}

Patologias em mucosa de esôfago podem cursar com sintomas de disfunção esofágica e alterações histopatológicas, que provocam queixa de dor em região epigástrica e retroesternal, sendo presença frequente nas consultas do gastroenterologista. É comum atribuir diagnóstico de doença do refluxo gastroesofágico, esofagite, gastrite e dispepsia. Em geral, a pesquisa da bactéria Helicobacter pylori (H.pylori, HP) tem sido solicitada. Entretanto, sabe-se que é na mucosa superficial do estômago onde a bactéria se instala provocando inflamação e, para se proteger do ácido gástrico, a bactéria produz amônia, o que possibilita a sua penetração na mucosa gástrica.

Segundo a OMS, a bactéria Helicobacter pylori acomete mais da metade da população mundial, apresenta uma incidência maior que 50\% em países subdesenvolvidos, além de ser considerada um carcinógeno de classe I para humanos. Autores citam que a presença de H. pylori causa maior probabilidade de carcinogênese na mucosa gástrica (Kaminski \& Kruel, 2001). Entretanto, 
a sua colonização também tem sido estudada em mucosa esofágica e na cavidade oral, questionando-se assim uma relação entre a bactéria H. pylori e a doença do refluxo gastroesofágico (DRGE), o esôfago de Barrett e esofagite (Lv, et al., 2019).

Sabe-se que a H. pylori apresenta uma relação com lesões gástricas e linfomas. Entretanto, associá-lo a lesões de esôfago tem sido motivo de estudos (Ribeiro, et al., 2016). Autores apontam que a positividade para a bactéria HP pode responder por queixas dispépticas (Andreolla, 2012). Segundo a literatura, a bactéria pode produzir uma resposta inflamatória diferente, quando comparada a indivíduos saudáveis, por considerar a ação desse patógeno na secreção ácida, e sua interferência na produção de quimiocinas e citocinas gástricas (Drossman, 2006). O achado histopatológico de inflamação crônica também tem sido atribuído como uma lesão comum nestes portadores de HP positivo, visto que, as células epiteliais gástricas quando estimuladas liberam citocinas pró-inflamatórias, consequentemente levando a uma maior presença local de células inflamatórias. Teorias tentam explicar a ação bacteriana como um fator provocador de lesões esofágicas. A composição microbiana do esôfago é diversificada, sendo portanto, um novo fator a ser considerado (Yang, et al., 2009; Guccione, et al., 2021). Este estudo se propôs a identificar a prevalência de achados histopatológicos de mucosa esofágica e correlacionar esses achados com a bactéria Helicobacter pylori.

\section{Metodologia}

Estudo transversal, retrospectivo, descritivo realizado de 2013 a 2018, na cidade de Aracaju, Sergipe, Brasil. O trabalho estatístico foi realizado por Íkaro Daniel de Carvalho Barreto, um dos autores deste estudo. Ele apresenta doutorado pelo Programa de Pós-Graduação em Biometria e Estatística Aplicada, pela UFRPE, em Recife, PE e PhD em Tecnologia Nuclear e Energetica, pela UFPE, em Recife, PE.

A amostra foi composta por 1.953 laudos histopatológicos de mucosa de esôfago e estômago do banco de dados de uma clínica privada de anatomia patológica, em Aracaju (SE), Brasil. Foram incluídas todas as lesões histopatológicas diagnosticadas em laudos de mucosa esofágica, portadores da bactéria helicobacter pylori e presença de atrofia gástrica. Foram excluídos laudos com identificação incompleta e diagnóstico inconclusivo. O instrumento de pesquisa foi composto pelas variáveis idade, sexo, procedência, presença ou não de H.pylori e identificação de lesões diagnosticadas em mucosa de transição esofagogástrica ou junto a mucosa glandular do tipo cárdico. Definiu-se nos laudos para o termo processo inflamatório: esofagite, processo inflamatório crônico em transição de mucosa escamosa e colunar de tipo cárdico / processo inflamatório junto a mucosa glandular do tipo cárdico.

Utilizou-se o software R Core Team 2021 (versão 4.1.0) e o nível de significância adotado foi de 5\%. As variáveiscategóricas foram descritas por frequência absoluta e relativa percentual e, as variáveis contínuas, por meio de mediana e intervalo quartil. A hipótese de independência entre variáveis categóricas testada por meio dos testes Qui-Quadrado de Pearson. A hipótese de aderência das variáveis contínuas à distribuição normal foi avaliada por meio do teste de Shapiro-Wilks. Como não foi confirmada, a hipótese de igualdade de duas medianas foi testada por meio do teste de Mann-Whitney. Foram estimados riscos relativos brutos e ajustados por meio da regressão de Poisson com erros padrões robustos simples e múltiplas. Para incluir variáveis no modelo ajustado foram utilizados os seguintes critérios: prevalência de ausentes inferior a 10\%, significância em teste de hipótese inferior ao nível de $20 \%$ e ausência de problemas de separação (caselas zeradas). Estudo aprovado pelo CEP/UNIT e parecer $n^{\circ} 1.246 .355$.

\section{Resultados}

Os laudos foram catalogados de acordo com os anos: 2013 (362), 2014 (371), 2015 (349), 2016 (237), 2017 (341), 2018 (293). A idade mediana foi de 44 anos (IIQ: 33 a 56), sendo 982 (50,3\%) do sexo feminino e 1505 (77,1\%) eram procedentes da capital. Do total de 1953 laudos analisados que apresentaram algum tipo de lesão em mucosa esofágica, 151 (7,7\%) eram 
Research, Society and Development, v. 11, n. 2, e33811225769, 2022

(CC BY 4.0) | ISSN 2525-3409 | DOI: http://dx.doi.org/10.33448/rsd-v11i2.25769

positivos para HP. Foram consideradas como lesões de mucosa esofágica mais prevalentes: esofagite/ processo inflamatório $1427(73,1 \%)$, metaplasia $548(28,1 \%)$ e esôfago de Barrett $133(6,8 \%)$. Do total das amostras $5(0,3 \%)$ laudos apresentaram diagnóstico de malignidade (adenocarcinoma) e 41 (2,3\%), atrofia gástrica. Não foi encontrado em nenhum laudo presença de hamartoma (Tabela 1). 
Tabela 1. Perfil dos portadores de lesões histopatológicas da mucosa esofágica. Aracaju, 2013-2018.

\begin{tabular}{|c|c|c|c|c|}
\hline & $\mathbf{n}$ & $\%$ & Mediana & IIQ \\
\hline \multicolumn{5}{|l|}{ Ano } \\
\hline 2013 & 362 & 18,5 & & \\
\hline 2014 & 371 & 19,0 & & \\
\hline 2015 & 349 & 17,9 & & \\
\hline 2016 & 237 & 12,1 & & \\
\hline 2017 & 341 & 17,5 & & \\
\hline 2018 & 293 & 15,0 & & \\
\hline Idade & & & 44 & $33-56$ \\
\hline \multicolumn{5}{|l|}{ Sexo } \\
\hline Feminino & 982 & 50,3 & & \\
\hline Masculino & 955 & 48,9 & & \\
\hline Ausente & 16 & 0,8 & & \\
\hline \multicolumn{5}{|l|}{ Procedência } \\
\hline Capital & 1505 & 77,1 & & \\
\hline Interior & 318 & 16,3 & & \\
\hline Demais Estados & 17 & 0,9 & & \\
\hline Ausente & 113 & 5,8 & & \\
\hline H. pylori & 151 & 7,7 & & \\
\hline Esôfago de Barret & 133 & 6,8 & & \\
\hline Esofagite/Processo Inflamatório & 1427 & 73,1 & & \\
\hline \multicolumn{5}{|l|}{ Classificação da Esofagite: } \\
\hline Ativa/Aguda & 91 & 9,3 & & \\
\hline Crônica & 885 & 90,7 & & \\
\hline Metaplasia & 548 & 28,1 & & \\
\hline \multicolumn{5}{|l|}{ Metaplasia Gástrica / } \\
\hline \multicolumn{5}{|l|}{ Glândulas tipo gástrica em córion/ } \\
\hline Ectopia Gástrica em esôfago & 414 & 75,5 & & \\
\hline Metaplasia Intestinal & 94 & 17,1 & & \\
\hline \multicolumn{5}{|l|}{ Metaplasia Intestinal Tipo } \\
\hline Focal & 35 & 6,4 & & \\
\hline Multifocal & 2 & 0,4 & & \\
\hline Difusa & 1 & 0,2 & & \\
\hline Não descrita & 1 & 0,2 & & \\
\hline Metaplasia Pancreática /Pâncreas Ectópico & 87 & 15,9 & & \\
\hline Heterotopia Gástrica & 6 & 0,3 & & \\
\hline Displasia & 21 & 1,1 & & \\
\hline \multicolumn{5}{|l|}{ Grau de Displasia } \\
\hline Baixo Grau & 12 & 57,1 & & \\
\hline Alto Grau & 9 & 42,9 & & \\
\hline Eosinófilos $>=15 \mathrm{eos} / \mathrm{CGA}$ & 78 & 4,0 & & \\
\hline Hiperplasia Epitelial & 61 & 3,1 & & \\
\hline Acantose Glicogênica & 31 & 1,6 & & \\
\hline Xantelasma & 3 & 0,2 & & \\
\hline Pólipos & 47 & 2,4 & & \\
\hline \multicolumn{5}{|l|}{ Localização/Região do Pólipo } \\
\hline Proximal & 4 & 8,5 & & \\
\hline Médio & 1 & 2,1 & & \\
\hline Distal & 11 & 23,4 & & \\
\hline Não Descrita & 31 & 66,0 & & \\
\hline \multicolumn{5}{|l|}{ Tipos de Pólipos } \\
\hline Adenomatoso & 2 & 4,3 & & \\
\hline $\begin{array}{l}\text { Inflamatório não ulcerado(erosão) / } \\
\text { Hiperplásico não ulcerado (erosão) }\end{array}$ & 12 & 25,5 & & \\
\hline Inflamatório ulcerado (erosão) / & & & & \\
\hline Hiperplásico ulcerado(erosão) & 1 & 2,1 & & \\
\hline Esofágico / & 24 & 51.1 & & \\
\hline Papiloma escamoso esofágico & 24 & 51,1 & & \\
\hline Não descrito & 8 & 17,0 & & \\
\hline Malignidade & 5 & 0,3 & & \\
\hline Atrofia Gástrica & 41 & 2,3 & & \\
\hline
\end{tabular}

Legenda: $\mathrm{n}$ - frequência absoluta. \% - frequência relativa percentual. IIQ - intervalo Interquartil. Fonte: Autores. 
Dentre aqueles que apresentaram positividade para H. pylori, a idade mediana foi de 42 anos (IIQ: 33 a 51), não houve prevalência quanto ao sexo e $120(80,5 \%)$ portadores eram procedentes da capital. Observou-se a prevalência de lesões como: esofagite/ processo inflamatório 97 (64,2\%), esôfago de Barrett 17 (11,3\%) e metaplasia 45 (29,8\%) sendo a metaplasia pancreática $16(10,6 \%)$, acantose glicogênica $9(6 \%)$ e atrofia gástrica 11(7,4\%) estatisticamente significativo (p<0,001). Apenas $2(1,3 \%)$ laudos apresentaram malignidade associados à presença de HP (Tabela 2).

Tabela 2. Correlação entre a presença da Helicobacter pylori e alterações histopatológicas de mucosa esofágica. Aracaju, 2013 - 2018.

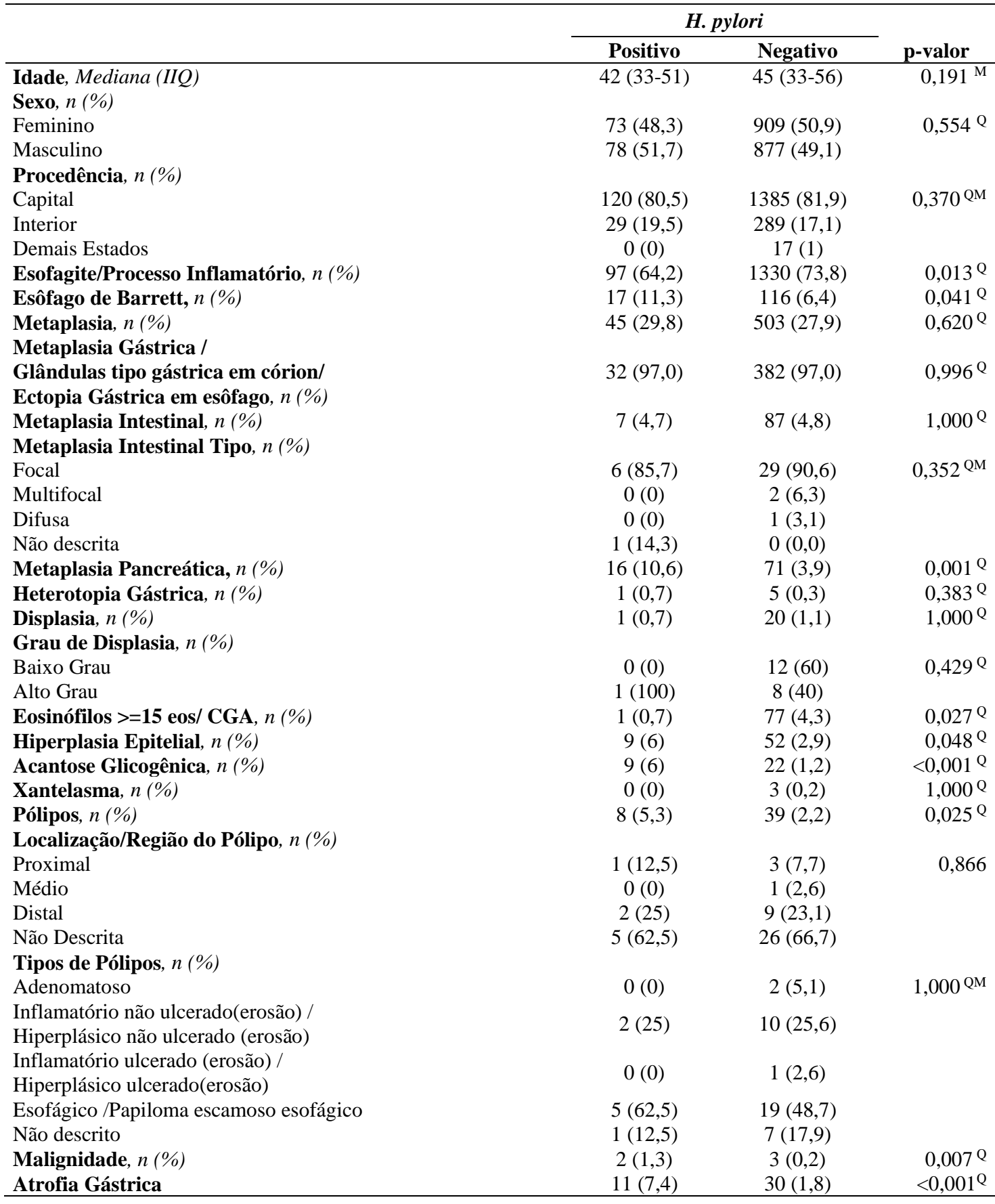

Legenda: $\mathrm{n}$ - frequência absoluta. \% - frequência relativa percentual. IIQ - intervalo interquartil. M - Teste de MannWhitney. Q - Teste Qui-Quadrado de Pearson. QM - Teste Qui-Quadrado de Pearson com simulações de MonteCarlo. Fonte: autores. 
Ao correlacionar a associação de $\mathrm{H}$. pylori com lesões de mucosa esofágica, observou-se que a presença das seguintes lesões na mucosa esofágica isoladamente podem determinar maior risco da presença da bactéria: esôfago de Barret (RR: 1,68 (IC95\%: 1,05-2,69)), Hiperplasia Epitelial (RR: 2,18 (IC95\%: 1,18-4,02)), Acantose Glicogênica (RR: 3,69 (IC95\%: 2,08-6,54)), Pólipos (RR: 2,17 (IC95\%: 1,14-4,16)), Malignidade (RR: 4,91 (IC95\%: 1,66-14,52)) e Atrofia Gástrica (RR: 3,44 (IC95\%: 2,02-5,84)). Além disso, a presença de Esofagite/Processo Inflamatório (RR: 0,68 (IC95\%: 0,49-0,94) pode determinar menor risco da presença da bactéria. Conjuntamente, temos que a presença de esôfago de Barret (RR: 1,83 (IC95\%: 1,13-2,95) p=0,013), Hiperplasia Epitelial (RR: 2,28 (IC95\%: 1,22-4,23) p=0,009), Acantose Glicogênica (RR: 3,62 (IC95\%: 1,98-6,62) p<0,001), Pólipos (RR: 2,19 (IC95\%: 1,09-4,39) p=0,028), Malignidade (RR: 3,65 (IC95\%: 1,27-10,52) p=0,016) e Atrofia Gástrica (RR: 3,12 (IC95\%: 1,84-5,29) $\mathrm{p}<0,001$ ) podem determinar maior risco da presença da bactéria (Tabela 3).

Tabela 3. Correlação entre lesões esofágicas e Helicobacter pylori. Aracaju, 2013-2018.

\begin{tabular}{lccc}
\hline & \multicolumn{2}{c}{ H. pylori } \\
\cline { 2 - 4 } & RR (IC95\%) & RRa (IC95\%) & p-valor \\
\hline Esôfago de Barret, $n(\%)$ & $1,68(1,05-2,69)$ & $1,83(1,13-2,95)$ & 0,013 \\
Esofagite/Processo Inflamatório, $n \%)$ & $0,68(0,49-0,94)$ & & \\
Eosinófilos $>=\mathbf{1 5}$ eos/ CGA, $n(\%)$ & $0,17(0,02-1,18)$ & & \\
Hiperplasia Epitelial, $n(\%)$ & $2,18(1,18-4,02)$ & $2,28(1,22-4,23)$ & 0,009 \\
Acantose Glicogênica, $n(\%)$ & $3,69(2,08-6,54)$ & $3,62(1,98-6,62)$ & $<0,001$ \\
Pólipos, $n(\%)$ & $2,17(1,14-4,16)$ & $2,19(1,09-4,39)$ & 0,028 \\
Malignidade, $n(\%)$ & $4,91(1,66-14,52)$ & $3,65(1,27-10,52)$ & 0,016 \\
Atrofia Gástrica & $3,44(2,02-5,84)$ & $3,12(1,84-5,29)$ & $<0,001$ \\
\hline
\end{tabular}

Legenda: RR - Risco Relativo. RRa - Risco Relativo Ajustado. IC95\% -Intervalo com 95\% de confiança. Fonte: Autores.

\section{Discussão}

A mucosa do esôfago pode apresentar várias reações teciduais como resposta a injúrias, seja de forma aguda ou crônica, por lesões degenerativas ou regenerativas, alterações devido a crescimento exagerado de células (hiperplasia) sem caráter de malignidade ou lesões de metaplasia, displasia e neoplasia.

A análise de laudos histopatológicos de mucosa esofágica como parte deste estudo, pôde identificar diversas lesões. A presença de esofagite $(73 \%)$, metaplasia $(28,1 \%)$ e esôfago de Barrett $(6,8 \%)$ foram lesões histopatológicas mais prevalentes dentre as lesões diagnosticadas em laudos de mucosa do esôfago.

A presença de eosinófilos maior ou igual a 15 eosinófilos por campo de grande aumento (CGA) foi em torno de 4\%. A esofagite eosinofílica é definida como uma doença crônica, esofágica, imunológica e/ou antígeno-mediada, caracterizada clinicamente por sintomas relacionados à disfunção esofágica e histologicamente por inflamação predominante de eosinófilos (da Silva Veiga, et al., 2017).

Quanto ao envolvimento de esofagite e a bactéria, ainda não há um consenso quanto à predisposição ou fator de risco (Ribeiro, et al., 2016). A literatura reforça que não há relação direta entre o H. pylori e o aparecimento da esofagite (Schutze, et al., 1995). Ainda assim, muitos outros estudos parecem relacionar de forma inversa a presença de HP com existência de esofagite de refluxo e DRGE (Na, et al., 2020).

Um estudo relatou a possibilidade de haver uma relação inversa entre a esofagite eosinofílica e a H. pylori (Ronkainen, et al., 2007). Autores relatam que a infecção por HP protege contra o desenvolvimento da esofagite erosiva (Na, et al., 2020). Em um estudo com 244 pacientes portadores de úlcera duodenal, a esofagite erosiva se desenvolvia com mais facilidade nos pacientes tratados por HP do que em pacientes com a infecção (Labenz, et al., 1997). Contudo, em uma metanálise mais recente, não houve relação estatisticamente significativa entre a esofagite de refluxo e a infecção pela HP (Saad, et al., 2012) 
Em outro estudo, foi concluído que o paciente com a bactéria H. pylori erradicada tem menos chance de desenvolver esofagite de refluxo do que naqueles pacientes que nunca foram infectados pela bactéria (Adachi, et al., 2019). Neste estudo, ao correlacionar a presença de HP e esofagite de terço distal do esôfago, identificou-se como sendo uma condição de menor risco.

Outro achado relevante neste estudo foi a presença de acantose glicogênica (AG) em associação a bactéria H. pylori ( $<<0,001)$. A acantose glicogênica é uma lesão polipóide, benigna, que geralmente é encontrada por meio da endoscopia digestiva alta, porém, a sua relevância clínica ainda não foi estabelecida. Histologicamente, a AG é caracterizada por placas multifocais com conteúdo de epitélio escamoso hiperplásico e de depósitos intracelulares de glicogênio (Y1lmaz, 2020). A associação entre AG e esofagite ainda é controversa (Stern, et al., 1980). Lesões como acantose glicogênica e pólipos apresentaram características similares a outro estudo. Esses autores identificaram que, lesão sem potencial de malignidade como a acantose glicogênica $(1,6 \%)$ e pólipos sem alterações displásicas $(2,4 \%)$ com prevalência na porção distal do esôfago $(23,4 \%)$ foram lesões identificadas em laudos histopatológicos (Schulz, et al., 2018).

De acordo com o Instituto Nacional do Câncer, a presença de displasia de baixo grau e de malignidade representaram em torno de 1,1 a 0,3\% respectivamente (INCA, 2019). A importância em identificar lesões desta natureza implica em diagnóstico precoce de câncer de esôfago, seja do carcinoma epidermóide escamoso, tipo mais comum, respondendo por 96\% dos casos, ou adenocarcinoma, o qual tem sido diagnosticado nas últimas décadas com mais frequência.

A presença de malignidade foi cerca de $0,3 \%$ do total da amostra e todos diagnosticados como adenocarcinomas de esôfago. Existem dois tipos de câncer de esôfago, sendo o carcinoma epidermóide escamoso o tipo mais comum, respondendo por $96 \%$ dos casos, sendo frequente na raça negra. Entretanto, o tipo adenocarcinoma, mais comum na raça branca, tem se revelado com mais frequência nas últimas décadas. Segundo o Instituto Nacional do Câncer, a estimativa de novos casos é de 11.390, sendo 8.690 homens e 2.700 mulheres (INCA, 2018). É considerado no Brasil como o sexto câncer mais comum entre os homens e o $15^{\circ}$ entre as mulheres. Na estatística mundial encontra-se no oitavo lugar em frequência e sua incidência é duas vezes maior quando comparado às mulheres (INCA, 2019).

O principal fator de risco para o desenvolvimento do adenocarcinoma esofágico é a doença do refluxo gastroesofágico (DRGE) e, consequentemente, o aparecimento do esôfago de Barrett (EB) (Screening \& Board, 2021). Estudos trazem uma relação inversamente proporcional entre a H. pylori e a incidência de adenocarcinoma esofágico (Souza, 2007). Os mecanismos conhecidos de proteção que a bactéria H. pylori exerce são centrados na diminuição da produção de ácido clorídrico concomitante à infecção da HP. O fato de identificar HP nos laudos de portadores de esôfago de Barrett, a mucosa gástrica foi avaliada, no sentido de identificar atrofia gástrica, o que poderia ser justificado pelo fato de que a colonização e inflamação pela HP, atrofia a mucosa gástrica levando a perda das células parietais e diminuição da secreção do ácido gástrico (Holleczek, et al., 2020). Em função dessa dinâmica funcional, o terço distal do esôfago não é agredido pelo ácido clorídrico e consequentemente menor incidência de alterações teciduais com capacidade carcinogênicas.

A associação entre HP e a incidência de carcinomas de células escamosas do esôfago (CECs) na junção esofagogástrica e no esôfago não foi observada segundo a literatura (Holleczek, et al., 2020). Ademais, a prevalência de malignidade observada nos laudos de amostras de esôfago de portadores de HP foi de 1,3\% (Xia \& Talley, 1998). Os dados deste estudo demonstraram similaridade com esses autores. Correlacionadas todas as lesões diagnosticadas com H. pylori positivo, a presença de metaplasia, acantose glicogênica e esofagite foram estatisticamente significativas. A literatura tem sido escassa quanto a correlação da bactéria Helicobacter pylori e as lesões comumente encontradas em terço inferior de esôfago, local que sofre a ação constante do ácido clorídrico. 


\section{Conclusão}

Achados de esofagite, metaplasia e esôfago de Barrett foram lesões histopatológicas mais prevalentes em laudos de mucosa do esôfago. Não houve prevalencia quanto ao sexo no total dos laudos analisados nem nos portadores da bactéria H. pylori. Todas as lesões diagnosticadas foram correlacionadas com a presença de H. pylori, entretanto, metaplasia pancreática e acantose glicogênica foram estatisticamente significativas, embora o diagnóstico de esofagite tenha sido percentualmente mais prevalente. Ao correlacionar a bactéria com as lesões esofágicas, identificou-se a esofagite como uma condição de menor risco. Entretanto, observou-se que o esôfago de Barrett, hiperplasia epitelial, acantose glicogênica, pólipos, malignidade e atrofia gástrica apresentaram uma tendência a determinar maior risco da presença da bactéria Helicobacter pylori. É necessário o incentivo para a elaboração de estudos posteriores sobre o tema, a fim de que a literatura se torne mais robusta e para que as questões levantadas neste estudo sejam trabalhadas sob vertentes variadas.

\section{Referências}

Adachi, K., Notsu, T., Mishiro, T., \& Kinoshita, Y. (2019). Long-term effect of Helicobacter pylori eradication on prevalence of reflux esophagitis. Journal of gastroenterology and hepatology, 34 (11), 1963-1967.

Guccione, C., Yadlapati, R., Shah, S., Knight, R., \& Curtius, K. (2021). Challenges in Determining the Role of Microbiome Evolution in Barrett's Esophagus and Progression to Esophageal Adenocarcinoma. Microorganisms, 9 (10), 2003.

Holleczek, B., Schöttker, B., \& Brenner, H. (2020). Helicobacter pylori infection, chronic atrophic gastritis and risk of stomach and esophagus cancer: Results from the prospective population-based ESTHER cohort study. International journal of cancer, 146 (10), $2773-2783$.

INCA. (2021). Câncer de esôfago. Instituto Nacional de Câncer. https://www.inca.gov.br/tipos-de-cancer/cancer-de-esofago.

INCA. (2021). Síntese de Resultados e Comentários. Instituto Nacional de Câncer. https://www.inca.gov.br/estimativa/sintese-de-resultados-e-comentarios.

Kaminski, E. D. M. F., \& Kruel, C. D. P. (2001). Carcinogênese gástrica. Revista HCPA. 21 (1), 86-97.

Labenz, J., Blum, A. L., Bayerdorffer, E., Meining, A., Stolte, M., \& Borsch, G. (1997). Curing Helicobacter pylori infection in patients with duodenal ulcer may provoke reflux esophagitis. Gastroenterology, 112 (5), 1442-1447.

Lv, J., Guo, L., Liu, J. J., Zhao, H. P., Zhang, J., \& Wang, J. H. (2019). Alteration of the esophageal microbiota in Barrett's esophagus and esophageal adenocarcinoma. World journal of gastroenterology, 25 (18), 2149.

Na H. K., Lee J. H., Park S. J., Park H. J., Kim S. O., Ahn J. Y., Kim D. H., Jung K. W., Choi K. D., Song H. J., Lee G. H., Jung H. Y. (2020). Effect of Helicobacter pylori eradication on reflux esophagitis and GERD symptoms after endoscopic resection of gastric neoplasm: a single-center prospective study. BMC Gastroenterol. 123 (20).

PDQ Screening and Prevention Editorial Board. (2021). Stomach (Gastric) Cancer Prevention (PDQ®): Health Professional Version. In PDQ Cancer Information Summaries. National Cancer Institute (US).

Ribeiro, P. F. S., Kubrusly, L. F., Nassif, P. A. N., Ribeiro, I. C. S., Bertoldi, A. D. S., \& Batistão, V. C. (2016). Relação entre graus de esofagite e o Helicobacter pylori. ABCD Arq Bras Cir Dig. 29 (3), 135-137.

Ronkainen, J., Talley, N. J., Aro, P., Storskrubb, T., Johansson, S. E., Lind, T., Bolling-Sternevald, E., Vieth, M., Stolte, M., Walker, M. M., \& Agréus, L. (2007).

Prevalence of oesophageal eosinophils and eosinophilic oesophagitis in adults: the population-based Kalixanda study. Gut, 56 (5), $615-620$.

Saad, A. M., Choudhary, A., \& Bechtold, M. L. (2012). Effect of Helicobacter pylori treatment on gastroesophageal reflux disease (GERD): meta-analysis of randomized controlled trials. Scandinavian journal of gastroenterology, 47 (2), 129-135.

Schulz, M. K., Biancardi, M. R., Fernandes, D., Almeida, L. Y. de, Bufalino, A., \& Leon, J. E. (2018). Glycogenic acanthosis on mouth clinically present as white plaque. RGO, Rev Gaúch Odontol. 66 (3), 274-277.

Schütze, K., Hentschel, E., Dragosics, B., \& Hirschl, A. M. (1995). Helicobacter pylori reinfection with identical organisms: transmission by the patients' spouses. Gut, $36(6), 831-833$.

Souza, R. C. A. D. (2007). Estudo da associação entre a esofagite erosiva e a infecção pelo Helicobacter pylori. Universidade Federal do Paraná, Curitiba.

Stern, Z., Sharon, P., Ligumsky, M., Levij, I. S., \& Rachmilewitz, D. (1980). Glycogenic acanthosis of the esophagus. A benign but confusing endoscopic lesion. The American journal of gastroenterology, 74 (3), 261-263.

Veiga, F. M. da S., Castro, A. P. B. M., Santos, C. de J. N. dos, Dorna, M. de B., \& Pastorino, A. C. (2017). Esofagite eosinofílica: um conceito em evolução? Arquivos de Asma, Alergia e Imunologia, 1 (4), 363-372. 
Research, Society and Development, v. 11, n. 2, e33811225769, 2022

(CC BY 4.0) | ISSN 2525-3409 | DOI: http://dx.doi.org/10.33448/rsd-v11i2.25769

Xia, H. H. X., \& Talley, N. J. (1998). Helicobacter pylori infection, reflux esophagitis, and atrophic gastritis: an unexplored triangle. The American journal of gastroenterology, 93 (3), 394-400.

Yang, L., Lu, X., Nossa, C. W., Francois, F., Peek, R. M., \& Pei, Z. (2009). Inflammation and intestinal metaplasia of the distal esophagus are associated with alterations in the microbiome. Gastroenterology, 137 (2), 588-597.

Yllmaz, N. (2020). The relationship between reflux symptoms and glycogenic acanthosis lesions of the oesophagus. Przeglad gastroenterologiczny, 15 (1), 39. 